\title{
It is safe and feasible to omit the chest tube postoperatively for selected patients receiving thoracoscopic pulmonary resection: a meta-analysis
}

\author{
Pengfei Li", Cheng Shen", Yanming Wu, Yutian Lai, Kun Zhou, Guowei Che \\ Department of Thoracic Surgery, West China Hospital, Sichuan University, Chengdu 610041, China \\ Contributions: (I) Conception and design: P Li, G Che; (II) Administrative support: None; (III) Provision of study materials or patients: None; (IV) \\ Collection and assembly of data: Y Wu, C Shen; (V) Data analysis and interpretation: Y Lai, K Zhou; (VI) Manuscript writing: All authors; (VII) \\ Final approval of manuscript: All authors. \\ \#These authors contributed equally to this work. \\ Correspondence to: Guowei Che. Department of Thoracic Surgery, West China Hospital, Sichuan University, Guoxuexiang No. 37, Chengdu 610041, \\ China. Email: guowei_che@yahoo.com.
}

Background: To access the feasibility and safety of no chest tube (NCT) placement after thoracoscopic pulmonary resection.

Methods: A comprehensive search of online databases (PubMed, Embase, Web of Science, and Cochrane library) was performed. Studies investigating the safety and feasibility of NCT compared with chest tube placement (CTP) after VATS pulmonary resection were eligible for our meta-analysis. Perioperative outcomes were extracted and synthesized. Specific subgroups (wedge resection) were examined. The methodological quality of the included articles was evaluated with the methodological index for nonrandomized studies (MINORS) tool.

Results: Analysis of 9 studies including a total of 918 patients was performed. Four hundred sixty-one patients underwent NCT and 457 patients underwent CTP. The length of stay (LOS) postoperatively in the NCT group was significant shorter than in the CTP group [standardized mean difference $(\mathrm{SMD})=$ $-0.80 ; 95 \%$ confidence interval (CI), -1.13 to $-0.47, \mathrm{P}=0.000]$. Patients in the NCT group experienced slighter pain than patients in the CTP group in postoperative day (POD) one (SMD $=-0.41 ; 95 \% \mathrm{CI}$, -0.75 to $-0.07, \mathrm{P}=0.02)$, and POD two $(\mathrm{SMD}=-0.41 ; 95 \% \mathrm{CI},-0.75$ to $-0.07, \mathrm{P}=0.02)$. While, there was no significant difference about the 30-day morbidity for patients who underwent NCT and CTP [relative ratio $(\mathrm{RR})=1.01 ; 95 \% \mathrm{CI}, 0.59-1.74, \mathrm{P}=0.04$ ) and the rate of re-intervention $(\mathrm{RR}=0.89 ; 95 \% \mathrm{CI}, 0.33-2.40$, $\mathrm{P}=0.57)$. No perioperative mortality was observed in both groups. The sensitivity analysis suggested that the relative effects between 2 groups have already stabilized. Subgroup analysis revealed an effect modification by operation approach regarding perioperative morbidity, but not for LOS.

Conclusions: This meta-analysis conforms that it is feasible and safe to omit chest tube after thoracoscopic pulmonary resection for patients carefully selected. Randomized controlled trails (RCTs) are urgently needed to verify this conclusion.

Keywords: Lung resection; meta-analysis; video-assisted thoracoscopic surgery (VATS); chest tube

Submitted Oct 28, 2017. Accepted for publication Mar 20, 2018.

doi: $10.21037 /$ jtd.2018.04.75

View this article at: http://dx.doi.org/10.21037/jtd.2018.04.75 


\section{Introduction}

Video-assisted thoracoscopic surgery (VATS) has been widely used by thoracic surgeons nowadays and shows comparable or even preferable results compared to thoracotomy lung resection $(1,2)$. This minimally invasive approach decreases the trauma of chest wall, and increase the course of postoperative rehabilitation. A chest tube is traditionally placed in the thoracic cavity for the fear of the occurrence of air leakage, bleeding and chylothorax after lung resection (3). However, the chest tube may increase chest pain and influence postoperative activity, which could prevent patients from rehabilitation (4). In contrast, the strategy of no chest tube (NCT) or early removal contributes to decrease the length of hospital stay and promote rehabilitation of patients $(5,6)$. As a method of fast-track rehabilitation, the NCT was omitting chest tube placement (CTP) postoperatively. For patients underwent this method, air leaks were tested by chest tubes in the early stage after surgery in the operation room, if no air leaks observed the chest tube would be removed.

Recently, several centers have published their experience of NCT after VATS lung resection (7-14). However, the efficacy and safety of NCT is still in debate. With the aim of determining whether NCT is comparable in short term outcomes with CTP, we conducted this meta-analysis to compare perioperative safety (30day morbidity, mortality, rate of reintervention), and postoperative efficacy (duration of hospitalization, pain scale) for VATS lung resection.

\section{Methods}

\section{Literature search and selection}

A systematic and comprehensive literature search of online databases PubMed, Embase (via OVID), Web of Science, and Cochrane library was performed to identify observational studies and randomized controlled trials (RCTs) published before August 2017 that examined the safety and efficacy of NCT and CTP. References were manually reviewed to identify additional available studies. Several search terms and related variants were used, including lung resection, VATS, chest tube, drainage, drain.

We evaluated all searched results according to the preferred reporting items for systematic reviews and metaanalyses (PRISMA) statement (15).

\section{Study inclusion/exclusion criteria}

Studies meeting the following criteria were included: patients underwent VATS pulmonary resection; RCTs or observational studies that investigated the safety and feasibility of NCT compared with CTP; at least 1 outcomes of interest were reported: perioperative safety (30-day morbidity, mortality, rate of re-intervention), and postoperative efficacy (duration of hospitalization, postoperative pain scale); only English studies were included. The selection of original studies was based on the process of viewing titles, abstracts, and full papers.

Exclusion criteria were defined as: noncomparative studies, review articles, abstracts, case reports, editorials, expert opinions, commentary articles, and letters were excluded.

\section{Data extraction and quality assessment}

Data were extracted independently by 2 investigators, and conflicts were adjudicated by a third investigator. For the selected studies, information on all available variables was extracted and entered into a Microsoft Excel database. The following outcomes were used to compare the two strategies: perioperative mortality, morbidity, length of hospitalization, rate of reintervention and postoperative pain score. Methodologic quality assessment of included studies was performed using the methodological index for non-randomized studies (MINORS) instrument (16). The MINORS contains 12 items, the first eight were specifically for non-comparative studies. The items are scored as: 0 (reported), 1 (reported but inadequate), 2 (reported and adequate), and a maximum score of 16 for non-comparative studies and a maximum score of 24 for comparative studies. Any disagreement was resolved via discussion among the authors until consensus was reached.

\section{Statistical analysis}

Relative ratio (RR) with $95 \%$ confidence interval (95\% CIs) was calculated for categorical outcomes (30-day morbidity and rate of reintervention). Standardized mean difference (SMD) with $95 \%$ CIs were calculated for continuous outcomes (duration of hospitalization, postoperative pain score). We used Cochran chi-square test and $\mathrm{I}^{2}$ to examine the heterogeneity among effect estimates. Statistical heterogeneity among studies was defined as $\mathrm{I}^{2}$ 


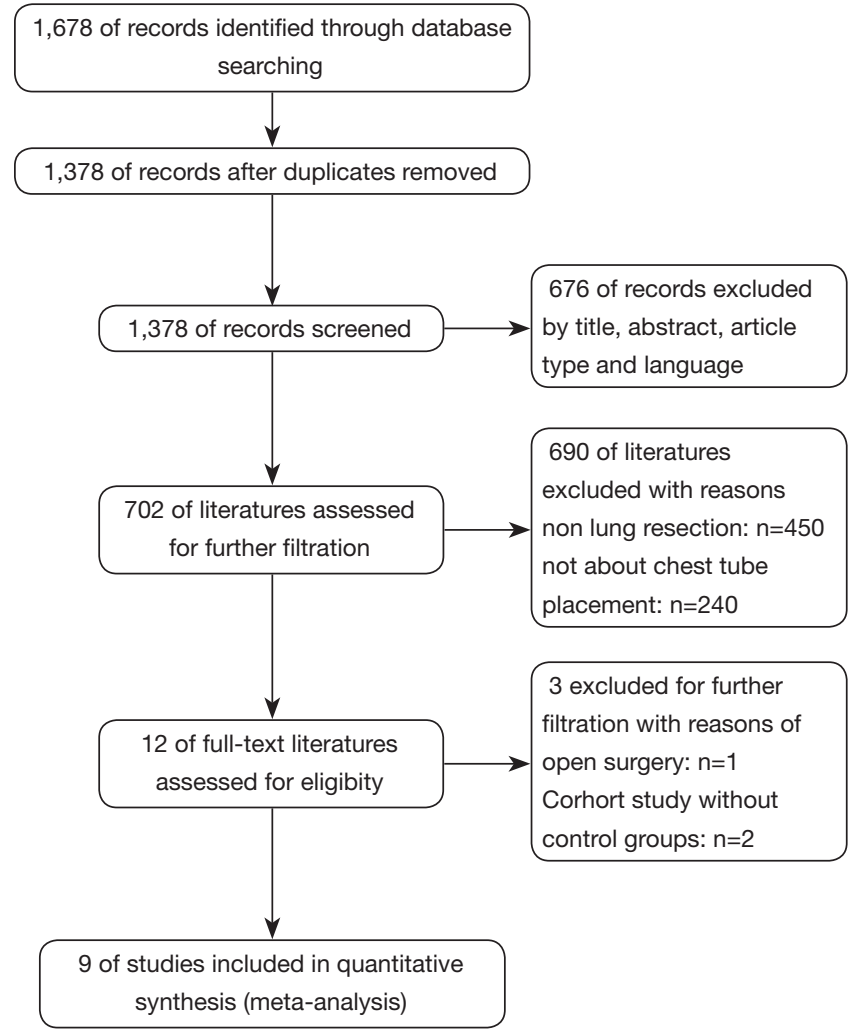

Figure 1 PRISMA flow diagram of the literature retrieval. PRISMA, preferred reporting items for systematic reviews and meta-analyses.

statistic greater than $50 \%$ (17). Fixed-effects model was preferred to random-effects model when there was no statistically significant heterogeneity (18). When necessary, mean and standard deviations (SDs) were estimated from the available median and CI or range (19). Inter-study heterogeneity was detected using the methods of subgroup analysis and sensitivity analysis. The subgroup analysis was based on surgical method of wedge resection and nonwedge resection and sensitivity analysis was by excluding each study individually from the meta-analysis. Statistical significance was taken as 2 -sided $(\mathrm{P}<0.05)$. The analysis was conducted with STATA 12.0 software (Stata Corporation, College Station, TX, USA).

\section{Results}

\section{Study selection}

In all, records were screened from the four previously mentioned online databases. A manual search and inspection of the reference lists and existing reviewed articles identified no additional relevant studies. After exclusion of duplicates, a total of 1,378 studies remained. Finally, 9 studies demonstrating the issue of omitting chest tube compared to CTP postoperatively were considered in this meta-analysis (Figure 1).

The studies were conducted in 4 different countries, during the period of 1997 to 2017. Three studies were prospective and the other six are retrospective observational studies, with no RCTs. In all, data from 918 patients were recorded, of which 461 patients underwent NCT and 457 patients underwent CTP. Two hundred twelve patients from two studies underwent thoracoscopic major lung resections such as lobectomy and segmentectomy, and 666 patients underwent thoracoscopic lung wedge resection, while 40 patients from one study underwent bullae resections.

\section{Quality assessment}

The quality assessment of the nine studies was performed using MINORS tool and the results of the quality assessment are reported in Table 1. Although all of the studies stated their main aims, the control groups were not equivalent to the study groups. Only three studies reported the consecutive inclusion of patients. None of studies reported the calculation of the sample size and unbiased assessment of the study endpoint (Table 1).

\section{Length of stay (LOS)}

The present meta-analysis indicates that the LOS postoperatively in the NCT group is significant shorter than in the CTP group (SMD $=-0.80 ; 95 \% \mathrm{CI},-1.13$ to $-0.47, \mathrm{P}=0.00)$, with a significant high heterogeneity $\left(\mathrm{I}^{2}=78 \%\right)$ (Table 2).

\section{Perioperative morbidity}

Meta-analysis demonstrated that the morbidity was similar between patients experienced NCT and CTP $(\mathrm{RR}=1.01$; 95\% CI, 0.59-1.74, $\mathrm{P}=0.04)$, with moderate heterogeneity $\left(\mathrm{I}^{2}=51.8 \%\right)$ (Table 2).

\section{Reintervention}

Reintervention was defined as reinsert of a chest tube or requiring thoracentesis postoperatively. There were no patients require a chest tube no thoracentesis in three 


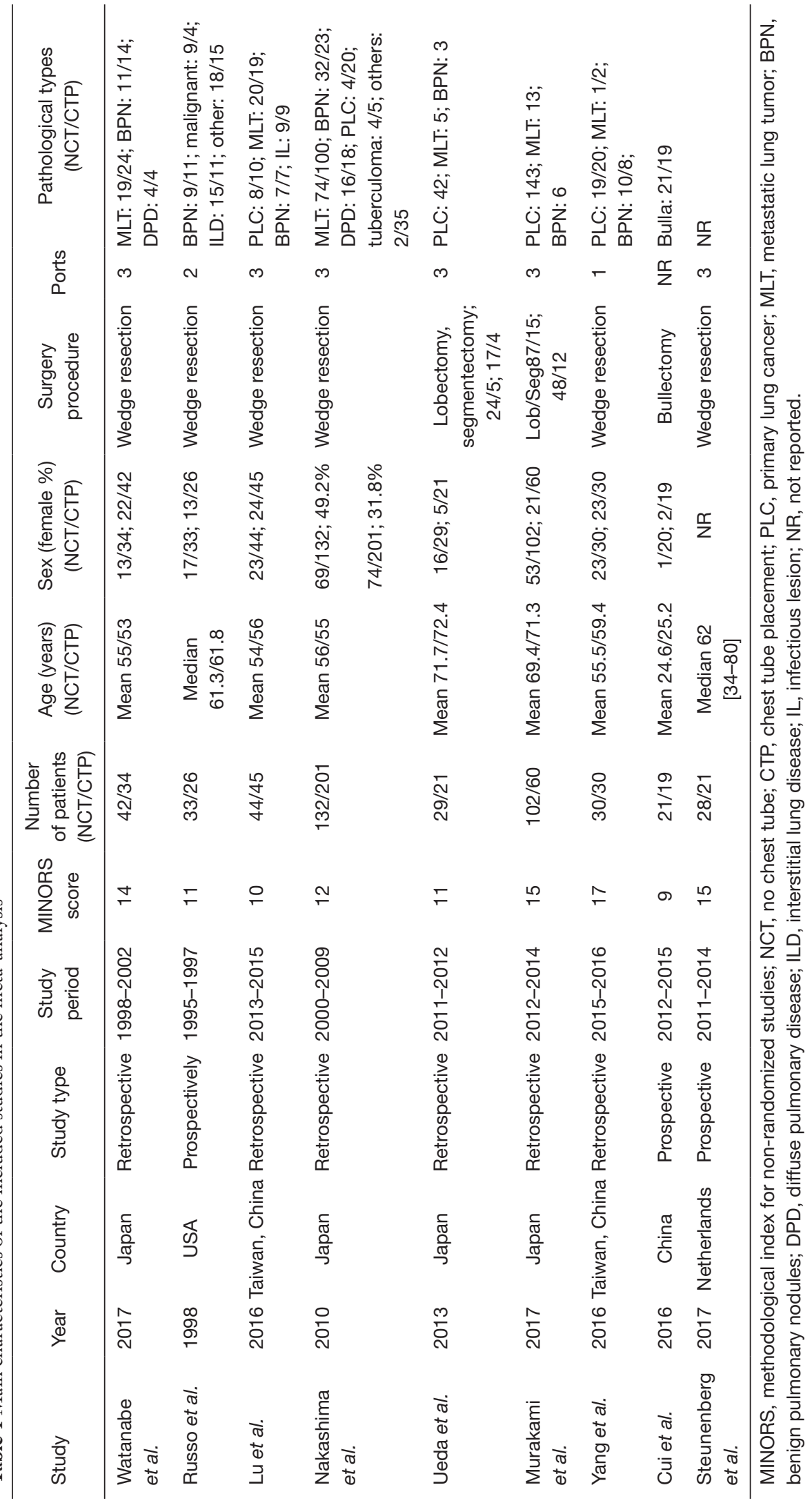


Table 2 Main outcomes of meta-analysis

\begin{tabular}{|c|c|c|c|c|c|c|c|}
\hline Analysis item & $\begin{array}{c}\text { No. of } \\
\text { studies }\end{array}$ & $\begin{array}{l}\text { Effects } \\
\text { model }\end{array}$ & RR/SMD (95\% Cl) & Significance & \multicolumn{3}{|c|}{ Heterogeneity test } \\
\hline Length of stay & 8 & Random & $-0.80(-1.13$ to -0.47$)$ & 0.00 & 31.80 & 78 & 0.00 \\
\hline Perioperative morbidity & 9 & Random & 1.01 (0.59 to 1.74$)$ & 0.97 & 16.60 & 51.8 & 0.04 \\
\hline Reintervention & 5 & Fixed & 0.89 (0.33 to 2.40$)$ & 0.82 & 2.92 & 0 & 0.57 \\
\hline Thoracocentesis & 3 & Fixed & 1.27 (0.25 to 6.55$)$ & 0.77 & 0.16 & 0 & 0.92 \\
\hline Pain scale, POD1 & 2 & Fixed & $-0.41(-0.75$ to -0.07$)$ & 0.02 & 0.33 & 0 & 0.56 \\
\hline Pain scale, POD2 & 2 & Fixed & $-0.41(-0.75$ to -0.07$)$ & 0.02 & 0.90 & 0 & 0.34 \\
\hline
\end{tabular}

$\mathrm{Cl}$, confidence interval; RR, relative risk; SMD, standardized mean difference; POD, postoperative day.

studies, so we included the remaining five studies into analysis. There was no significant difference in the rate of reintervention $(\mathrm{RR}=0.89 ; 95 \% \mathrm{CI}, 0.33-2.40, \mathrm{P}=0.57$ ), without heterogeneity $\left(\mathrm{I}^{2}=0\right)$. We did not observe significant difference about the rate of treat chest tube reinsertion $(\mathrm{RR}=1.00 ; 95 \% \mathrm{CI}, 0.39-2.54, \mathrm{P}=0.33)$, with slightly heterogeneity $\left(\mathrm{I}^{2}=14.1 \%\right)$. And there was no significant difference about the rate of thoracentesis in the NCT group compared with the CTP group (RR $=1.27 ; 95 \% \mathrm{CI}$, $0.25-6.55, \mathrm{P}=0.92)$, without heterogeneity $\left(\mathrm{I}^{2}=0\right)$ (Table 2$)$.

\section{Postoperative pain scale}

Available visual analogue scale (VAS) data could be extracted from two studies into our meta-analysis, and the pain scale in the NCT group was significant lower than in the CTP group in POD one (SMD $=-0.41 ; 95 \% \mathrm{CI},-0.75$ to -0.07 , Sig. =0.02), without heterogeneity $\left(\mathrm{I}^{2}=0, \mathrm{P}=0.56\right)$, and POD two (SMD $=-0.41 ; 95 \% \mathrm{CI},-0.75$ to -0.07 , Sig. $=0.02$ ), without heterogeneity $\left(\mathrm{I}^{2}=0, \mathrm{P}=0.34\right)$ (Table 2).

\section{Subgroup analysis}

To explore potential sources of statistical heterogeneity among the studies and to assess the stability of results, we conducted stratified analyses. Outcomes regarding the LOS and perioperative morbidity were separated into two groups by whether the operation approach was VATS wedge resection or other approaches (VATS major lung resection and bullectomy). Comparing with the overall outcomes, the heterogeneity significantly decreased when we preformed the subgroup analysis by operation approach about perioperative morbidity but not for LOS (Figure 2).

\section{Sensitivity analysis}

A sensitivity analysis was performed by excluding the studies with the lowest-quality score, none of the outcome data from the included studies was found to be out of the estimated range in each quantitative synthesis (Figure 3).

\section{Discussion}

Compared with conventional open thoracotomy, VATS relates to reduced pain, lesser short-term postoperative pain, less intraoperative blood loss during surgery, and shorter hospital stay and faster return to full activity (1). Traditionally, a chest tube would be placed in patients undergoing pulmonary surgery in order to drain air leakages and to observe whether there was flood bleeding or chylothorax $(3,20)$. However, CTP may enhance postoperative pain, deteriorate the ventilation capacity and increase the risk of infectious complication such as pneumonia and empyema, and prolong LOS. Therefore, thoracic surgeons have attempted to control air leakages detected intraoperatively in order to allow chest tubes to be removed early in the postoperative course (20-23). A number of clinical trials have been conducted to explore the feasibility and safety of omitting chest tube after pulmonary surgery, and found that NCT after pulmonary does not increase the morbidity and mortality, and meanwhile decrease the pain scale postoperatively. Some institutions have made efforts to explore the benefits of NCT especially for small pulmonary nodules underwent VATS wedge 


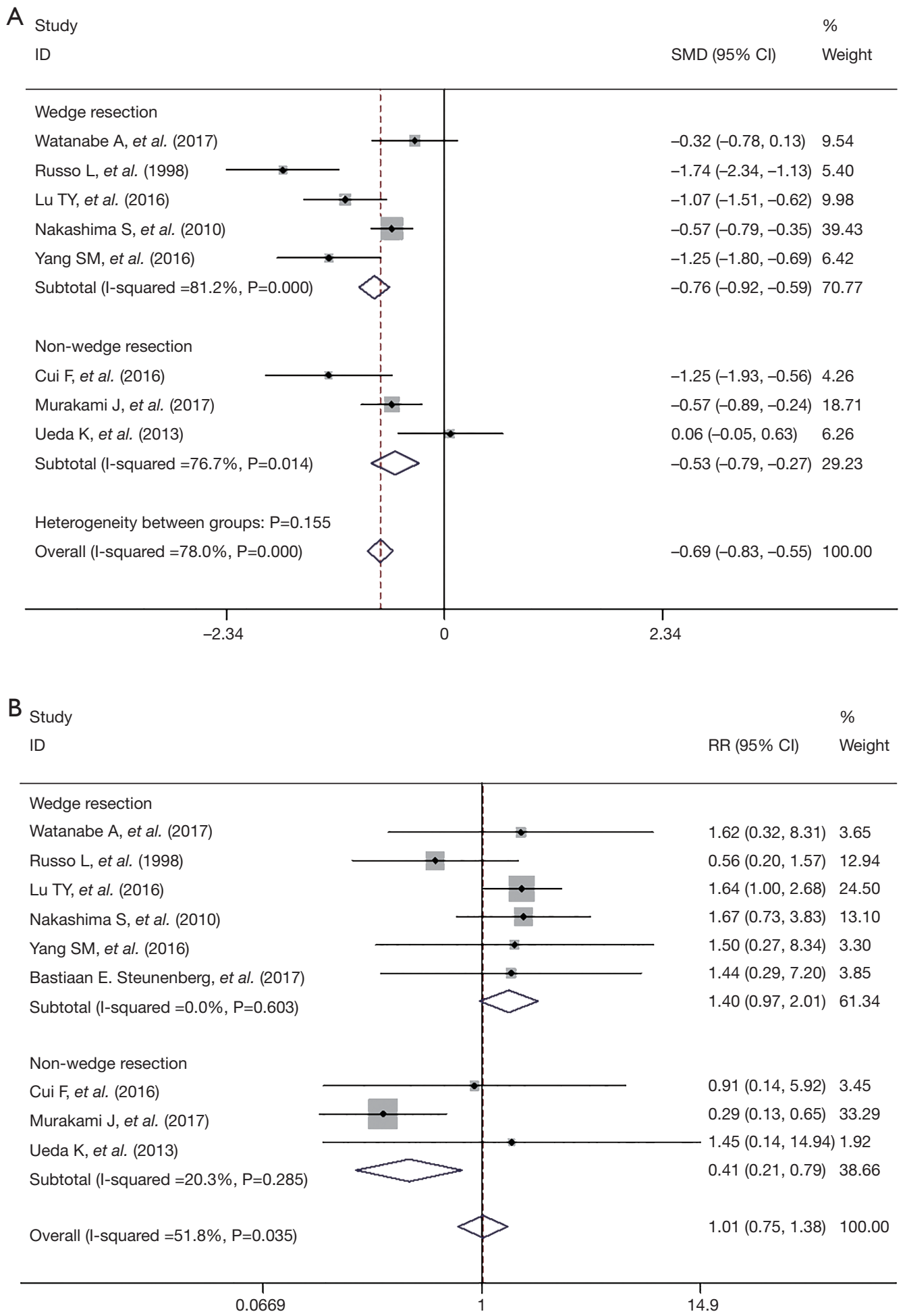

Figure 2 Subgroup analysis of (A) the length of hospital stay; (B) morbidity. CI, confidence interval. 

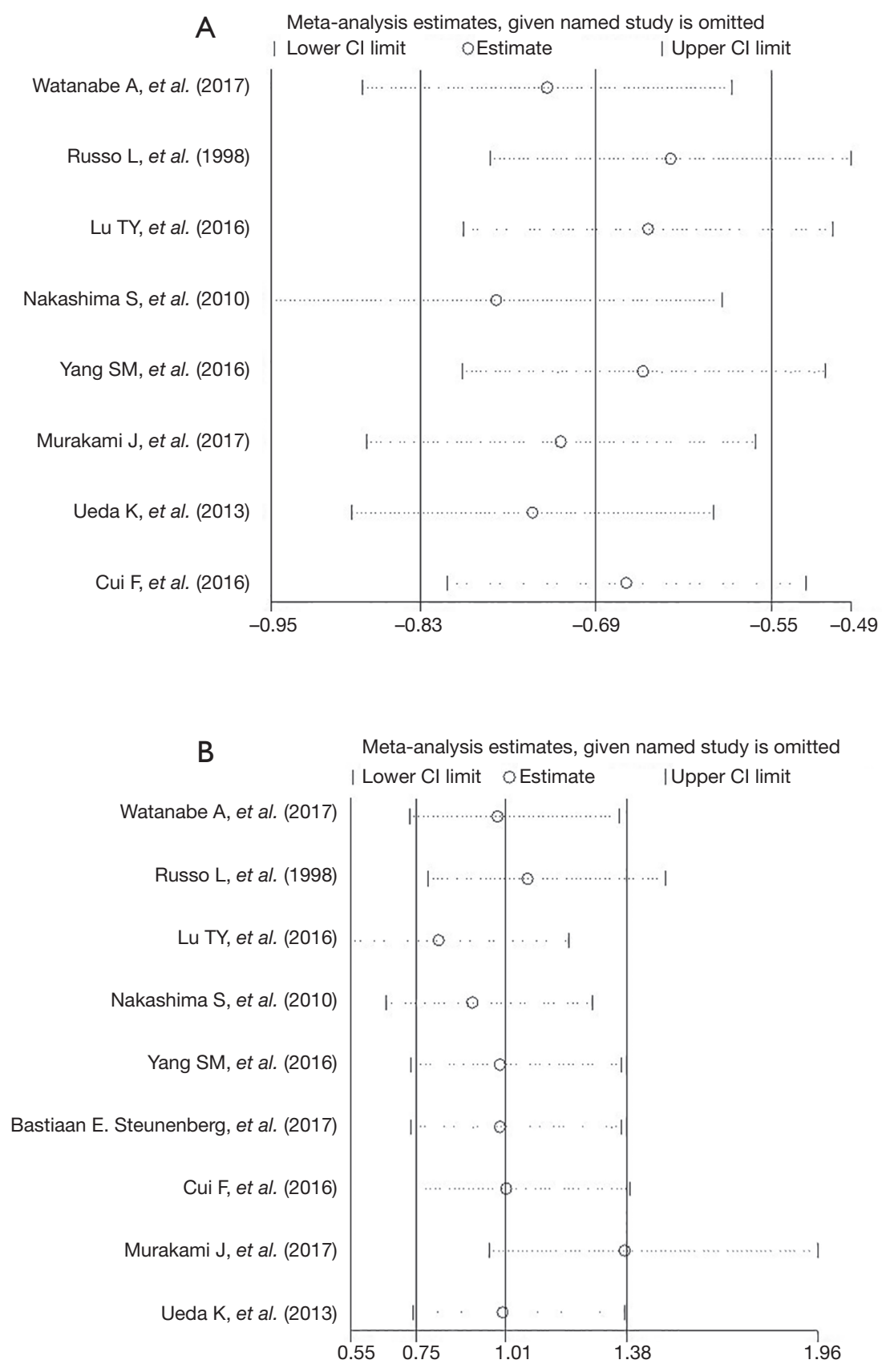

Figure 3 Sensitivity analysis of (A) the length of hospital stay; (B) morbidity. CI, confidence interval.

resections $(5-7,9,10,24)$. At the same time, a few studies set out to explore the feasibility of NCT for patients experiencing VATS major lung resection $(8,11,25)$.

So the present meta-analysis includes comparative studies of the safety and feasibility of NCT and CTP for patients underwent pulmonary resection. As described above, the majority of the studies were of moderate to high quality although none of the studies were RCTs. Three of the studies included was of prospective character and the other 6 studies were retrospective cohort studies.

The 30-day morbidity was similar between the two groups about NCT and CTP (RR $=1.01 ; 95 \% \mathrm{CI}$, 
0.59-1.74), which indicated that omitting chest tube postoperative did not increase the rate of morbidity. As for perioperative mortality, no patient died in either group, so we failed to synthesis the data about mortality. On the other side, we could say that omitting chest tube did not increase the rate of perioperative mortality.

In one recently published cohort study the authors assess the feasibility of avoiding chest drains following uniportal VATS wedge resection for pulmonary nodules. Totally 49 patients under 51 VATS wedge resections, and no patients required reinsertion of a chest tube, with no complications on 30-day follow-up (12). Li et al. performed a prospective study to evaluate the feasibility of a new tubeless (spontaneous ventilation without tracheal intubation, urinary catheterization, and no postoperative chest drain placement) approach to perform VATS for pulmonary nodules less than $2 \mathrm{~cm}$ in diameter. Finally, 34 patients were included in this research, 26 patients were discharged within $24 \mathrm{~h}$ after surgery and the remaining 8 patients were discharged on the second day. None of the patients needed re-invention with chest drainage or urinary catheterization even after discharge (24), which indicated that NCT after VATS pulmonary wedge resection is safe for selected patients.

Postoperative pain would prevent patients to cough effectively and this may have an impact on sputum excretion, which would probably increase the risk of atelectasis and pneumonia. On the other hand, postoperative pain may prevent patients from getting out of bed early, thus affecting the recovery of functions $(4,23)$. Furthermore, antalgesic dosage raises in order to relieve pain. Thoracic surgeons have tried amounts of methods to reduce postoperative pain and have achieved much progress such as reducing the number of chest tubes, using more flexible and slender chest tubes $(21,26,27)$. Five of the nine included studies reported postoperative pain scale measured in the VAS $(6,8,10,11,13)$ and three study reported the dosage of antalgesic $(5,9,13)$. Patients in the NCT group have lighter pain in POD1 and used less antalgesic. However, available data could be extracted from only two studies, and the VAS was significant lower for patients in the NCT group in POD1 (SMD = $-0.41 ; 95 \%$ CI, -1.75 to -0.01 ) and POD2 (SMD $=-0.41$; $95 \%$ CI, -0.53 to -1.75 ).

For patients that were classified into the NCT group, the primary question that we must consider is whether this strategy would increase the rate of pneumothorax and increase the rate of reintervention. After data synthesis, we found that there was no significant difference regarding the rate of re-intervention ( $\mathrm{RR}=0.89 ; 95 \%$ CI, 0.33-2.40). Meanwhile, no significant difference was discovered when we independently synthesize the data of chest tube reinsertion ( $\mathrm{RR}=1.00 ; 95 \% \mathrm{CI}, 0.39-2.54$ ). Likewise, it revealed no significant difference about the rate of thoracentesis for postoperative pneumothorax and pleural effusion ( $\mathrm{RR}=1.27$; 95\% CI, 0.25-6.55). Another important dangerous risk associated with this management is silent massive hemorrhage in the thoracic cavity after the operation. To avoid this situation, other monitor methods such as arterial blood pressure and heart rate should be closely monitored. Once consistent arterial blood pressure decreasing and heart rate increasing occurred, hemorrhage in the thoracic cavity should be considered and reoperation should be more aggressive.

To ensure the security of this strategy, patients assigned into the NCT group should meet some criteria. A sealing test to test air leaks is inevitable during the operation in the operating room before the holes were totally closed. If no air leaks were observed then the patients would be assigned to the NCT group, otherwise to the CTP group. Six of nine studies adopt water seal test to check air leakage $(6-10,13)$ and one study adopt the suction-induced air leakage test (11), because they suppose ipsilateral remaining lung is not fully inflated during the water-seal test, so some leaks may be failed to be detected during the water seal test. One study published in 1998 used an otherwise early removal approach (5). Patients assigned to the NCT group experienced early chest tube removal within 90 min of the surgical procedure in the recovery room. Although this was not a strict NCT strategy, we include this study into our meta-analysis for that traditional chest tube management tends to keep the chest tube inserted for at least $24 \mathrm{~h}$.

We acknowledge several limitations to this meta-analysis. First of all, none of the included studies were RCTs, with a greater risk of potential selection and reporting bias. Second, different surgical methods including wedge resection, major pulmonary resection and bullae resections and different types of pathology were included in this metaanalysis, which inevitably increase the clinical heterogeneity. Though the rate of postoperative re-intervention had low heterogeneity, the 30-day morbidity and postoperative LOS showed relative high heterogeneity. Factors that could potentially explain the heterogeneity include the levels of different surgeons' experience, different standard to diagnose perioperative morbidity, different medical insurance of regions and countries. For example, the health care system is unique in Japanese that patients are 
permitted to stay a relatively long time in the hospital, even though they could be discharged earlier. Third, all of the studies included had their own criteria to select patients into the NCT groups, this may give rise to different baseline characteristic of the two groups and a high clinical heterogeneity. Fourth, the majority of included studies reported an insufficient follow-up period.

The criteria to select patients into the NCT group are still in the air although there were consistent clauses in the included studies. The absence of air leaks during an intraoperative sealing test, the absence of bullous or emphysematous changes in the lung, the absence of dense pleural adhesion were considered important factors to select patients.

\section{Conclusions}

This meta-analysis reveals that the NCT strategy is feasible and safe for selected patients scheduled for VATS pulmonary surgery. Patients in the NCT group were found to have similar 30-day morbidity and shorter postoperative LOS and patients experienced lighter pain postoperatively. These findings seem to support the use of NCT strategy, however patients should be carefully selected to meet specific criteria. Further studies especially RCTs and large scale cohort studies are urgently needed to evaluate the feasibility and safety of NCT.

\section{Acknowledgements}

We greatly appreciate the assistance of the staff of the Department of Thoracic Surgery, West-China Hospital, Sichuan University, and thank them for their efforts.

Funding: This study was supported by Foundation of Science and Technology support plan. Department of Sichuan Province (No. 2015SZ0158).

\section{Footnote}

Conflicts of Interest: The authors have no conflicts of interest to declare.

\section{References}

1. Hartwig MG, D'Amico TA. Thoracoscopic lobectomy: the gold standard for early-stage lung cancer? Ann Thorac Surg 2010;89:S2098-101.

2. Cao C, Zhu ZH, Yan TD, et al. Video-assisted thoracic surgery versus open thoracotomy for non-small-cell lung cancer: a propensity score analysis based on a multi-institutional registry. Eur J Cardiothorac Surg 2013;44:849-54.

3. Miller KS, Sahn SA. Chest tubes. Indications, technique, management and complications. Chest 1987;91:258-64.

4. Refai M, Brunelli A, Salati M, et al. The impact of chest tube removal on pain and pulmonary function after pulmonary resection. Eur J Cardiothorac Surg 2012;41:820-2.

5. Russo L, Wiechmann RJ, Magovern JA, et al. Early chest tube removal after video-assisted thoracoscopic wedge resection of the lung. Ann Thorac Surg 1998;66:1751-4.

6. Watanabe A, Watanabe T, Ohsawa H, et al. Avoiding chest tube placement after video-assisted thoracoscopic wedge resection of the lung. Eur J Cardiothorac Surg 2004;25:872-6.

7. Nakashima S, Watanabe A, Mishina T, et al. Feasibility and safety of postoperative management without chest tube placement afterthoracoscopic wedge resection of the lung. Surg Today 2011;41:774-9.

8. Ueda K, Hayashi M, Tanaka T, et al. Omitting chest tube drainage after thoracoscopic major lung resection. Eur J Cardiothorac Surg 2013;44:225-9.

9. Lu TY, Chen JX, Chen PR, et al. Evaluation of the necessity for chest drain placement following thoracoscopic wedge resection. Surg Today 2017;47:606-10.

10. Yang SM, Wang ML, Hung MH, et al. Tubeless Uniportal Thoracoscopic Wedge Resection for Peripheral Lung Nodules. Ann Thorac Surg 2017;103:462-8.

11. Murakami J, Ueda K, Tanaka T, et al. The Validation of a No-Drain Policy After Thoracoscopic Major Lung Resection. Ann Thorac Surg 2017;104:1005-11.

12. Holbek BL, Hansen HJ, Kehlet H, et al. Thoracoscopic pulmonary wedge resection without post-operative chest drain: an observational study. Gen Thorac Cardiovasc Surg 2016;64:612-7.

13. Cui F, Liu J, Li S, et al. Tubeless video-assisted thoracoscopic surgery (VATS) under non-intubated, intravenous anesthesia with spontaneous ventilation and no placement of chest tube postoperatively. J Thorac Dis 2016;8:2226-32.

14. Steunenberg BE, Aerts BA, de Groot HG, et al. Is it still necessary to leave a chest tube after video-assisted pulmonary wedge resection? Indian J Thorac Cardiovasc Surg 2017;33:132-6.

15. Knobloch K, Yoon U, Vogt PM. Preferred reporting items for systematic reviews and meta-analyses (PRISMA) 
statement and publication bias. J Craniomaxillofac Surg 2011;39:91-2.

16. Slim K, Nini E, Forestier D, et al. Methodological index for non-randomized studies (minors): development and validation of a new instrument. ANZ J Surg 2003;73:712-6.

17. DerSimonian R, Laird N. Meta-analysis in clinical trials. Control Clinl Trials 1986;7:177-88.

18. Hozo SP, Djulbegovic B, Hozo I. Estimating the mean and variance from the median, range, and the size of a sample. BMC Med Res Methodol 2005;5:13.

19. Deng B, Qian K, Zhou JH, et al. Optimization of Chest Tube Management to Expedite Rehabilitation of Lung Cancer Patients AfterVideo-Assisted Thoracic Surgery: A Meta-Analysis and Systematic Review. World J Surg 2017;41:2039-45.

20. Gómez-Caro A, Roca MJ, Torres J, et al. Successful use of a single chest drain post lobectomy instead of two classical drains: a randomized study. Eur J Cardiothorac Surg 2006;29:562-6.

21. Okur E, Baysungur V, Tezel C, et al. Comparison of the single or double chest tube applications after pulmonary lobectomies. Eur J Cardiothorac Surg 2009;35:32-5.

Cite this article as: Li P, Shen C, Wu Y, Lai Y, Zhou K, Che G. It is safe and feasible to omit the chest tube postoperatively for selected patients receiving thoracoscopic pulmonary resection: a meta-analysis. J Thorac Dis 2018;10(5):2712-2721. doi: 10.21037/ jtd.2018.04.75
22. Nakamura H, Taniguchi Y, Miwa K, et al. The 19Fr Blake drain versus the $28 \mathrm{Fr}$ conventional drain after a lobectomy for lung cancer. Thorac Cardiovasc Surg 2009;57:107-9.

23. Li S, Jiang L, Ang KL, et al. New tubeless video-assisted thoracoscopic surgery for small pulmonary nodules. Eur J Cardiothorac Surg 2017;51:689-93.

24. Göttgens KW, Siebenga J, Belgers EH, et al. Early removal of the chest tube after complete video-assisted thoracoscopic lobectomies. Eur J Cardiothorac Surg 2011;39:575-8.

25. Gómez-Caro A, Roca MJ, Torres J, et al. Successful use of a single chest drain postlobectomy instead of two classical drains: a randomized study. Eur J Cardiothorac Surg 2006;29:562-6.

26. Ishikura H, Kimura S. The use of flexible silastic drains after chest surgery: novel thoracic drainage. Ann Thorac Surg 2006;81:331-3.

27. Coughlin SM, Emmerton-Coughlin HM, Malthaner R. Management of chest tubes after pulmonary resection:a systematic review and meta-analysis. Can J Surg 2012;55:264-70. 